\title{
An Improved Equation of State for Gases
}

\author{
JOSEPH J. MARTIN, RAJENDRA M. KAPOOR, and NOEL DE NEVERS, University of Michigan, Ann Arbor, Michigan
}

Based on properties which are characteristic of all gases, modifications are developed for an equation of state previously proposed in this journal (1). A specific application of the modified equation is made for the PVT data on carbon dioxide, and considerable improvement over the original equation is shown for densities in the neighborhood of 1.4 times the critical density. The new equation differs from the old equation only by the presence of the $A_{5}$ and $C_{5}$ terms, these having originally been taken to be zero.

In an earlier paper the general PVT characteristics of pure gases and liquids were presented by Martin and Hou (1). The hypothesis was advanced that complete representation of the $P V T$ behavior of any pure gas requires a knowledge of the critical temperature, pressure, and volume and of the slope of the vaporpressure curve at the critical point. Based on this hypothesis an equation of state of the following form was developed:

$$
P=\sum_{i=1}^{i=5} \frac{f_{i}}{(V-b)^{i}}
$$

In this expression the $f_{i}$ 's are temperature functions of the kind

$f_{i}=A_{i}+B_{i} T$

$$
+C_{i} \exp (-k T / T c)
$$

Although it was desirable to evaluate all the constants, it did not prove possible at the time; therefore $A_{1}, C_{1}, B_{4}, C_{4}, A_{5}$, and $C_{5}$ were set equal to zero, and $B_{1}$ was set equal to $R$ so that proper behavior would be obtained at infinite attenuation (that is, ideal-gas state). The expanded form of the original equation is

$$
\begin{aligned}
P & =\frac{R T}{V-b} \\
& +\frac{A_{2}+B_{2}+C_{2} \exp (-k T / T c)}{(V-b)^{2}} \\
& +\frac{A_{3}+B_{3} T+C_{3} \exp (-k T / T c)}{(V-b)^{3}} \\
& +\frac{A_{4}}{(V-b)^{4}}+\frac{B_{5} T}{(V-b)^{5}}
\end{aligned}
$$

and the constants are evaluated from formulas derived from the general PVT characteristics and the basic hypothesis. The derivations of these formulas are presented in the original paper (1).

\section{THE MODIFICATION}

Since its original development six years ago, the equation of state has been applied to a number of compounds. In some cases only the minimum four facts $\left(P_{c}, T_{c}, V_{c}\right.$, and $m$, the slope of the vapor-pressure curve at the critical) were employed to get the equation constants. In other cases the equation was fitted to the actual data by adjusting constants. To understand the new modification, it is desirable to know how the equation is fitted to the data for a given gas. When one refers to an isometric plot such as shown in the accompanying figure, the first step consists in obtaining the temperature functions along the critical isotherm. In this procedure the data are fitted with extremely high precision at the critical temperature to a density of about 1.5 times the critical density. The critical isometric is then fitted exactly from the critical point to the highest temperature to which it extends as a straight line. For all ordinary substances this is the highest temperature encountered in practical use.* Next, by means of the Boyle point temperature, the data are fitted at high temperature and low density. By means of the $T^{\prime}$ temperature the data are fitted at low temperature and low density.

For densities a little over the critical and temperatures above the critical there was no actual fitting operation even when data were available. Comparison of the equation with the experimental data in this region, which is circled in the figure, showed, however, that the isometrics predicted by the equation curved upward a little too much. This was true for a wide variety of gases. To correct this curvature of the higher density isometrics, recourse was made to two additional properties that are common to all gases. As pointed out in the original paper, the isometrics at about 1.5 to 2.0 times the critical density tend to be straight, just as at the critical density. Mathematically this means that

$\left(d^{2} P / d T^{2}\right)_{V}=0$ at $V=V_{c} / n$ (4) where $n$ lies between 1.5 and 2.0. The slope of this isometric is from three to eight times the slope of the critical isometric, a condition which may be stated as

$$
(d P / d T)_{V}\left(\text { at } V=V_{c} / n\right)
$$$$
=y(d P / d T)_{V}\left(\text { at } V=V_{c}\right)=y m
$$

From Equations (4) and (5) it is possible to evaluate two more constants in the general equation of state. Because the equation as originally developed satisfactorily represents the data at low densities, it was desirable to make modifications which would have little effect on the equation at low densities. New constants therefore were placed in the $f_{5} /(V-b)^{5}$ term, which rapidly approaches zero as $V$ becomes large. In the original equation $A_{6}$ and $C_{5}$ were

*For low-boiling-point gases, such as hydrogen and helium, the critical isometrics curve downward at the highest temperatures. This downward curvature occurs only at high reduced temperatures which are not encountered with ordinary compounds. To fit the data for low-boiling-point gases, it is necessary arbitrarily set equal to zero; in the modified form they have been given finite values determined from relations (4) and (5).

The proposed modification does not affect the formulas for the evaluation of the $f_{i}\left(T_{c}\right)$ or the formulas for $A_{2}, B_{2}, C_{2}$, and $A_{4}$; it does necessitate the formulation of new equations for the constants $A_{3}, B_{3}$, $C_{3}, A_{5}, B_{5}$, and $C_{5}$. The formulas for these, derived from the conditions previously used to solve for $A_{2}, B_{3}, C_{2}$, and $B_{5}$ plus the two new conditions, are outlined as follows:

From

$\left(d^{2} P / d T^{2}\right)_{V}=0$ at the critical density (6)

$\left(d^{2} P / d T^{2}\right)_{V}=0$

at $n$ times the critical density.

one may obtain by differentiating the proposed equation

$$
\begin{array}{r}
C_{2} /(V-b)^{2}+C_{3} /(V-b)^{3} \\
+C_{5} /(V-b)^{5}=0
\end{array}
$$

at $V=V_{c}$ and at $V=V_{c} / n$. Eliminating $C_{5}$ and solving for $C_{3}$ in terms of $C_{2}$ (which is already known), one gets

$$
C_{3}=\frac{C_{2}\left[\left(V_{c}-b\right)^{3}-\left(V_{c} / n-b\right)^{3}\right]}{\left(V_{c} / n-b\right)^{2}-\left(V_{c}-b\right)^{2}}
$$

and

$$
\begin{aligned}
C_{5}=-C_{2}\left(V_{c}\right. & -b)^{3} \\
& -C_{3}\left(V_{c}-b\right)^{2}
\end{aligned}
$$

The remaining constants to be evaluated are $A_{3}, B_{3}, A_{5}$ and $B_{5}$. The two $B^{\prime} \mathrm{s}$ are solved for by means of the conditions

$(d P / d T)_{V}=m$ at $V=V_{0}$

and

$$
(d P / d T)_{V}=y m \text { at } V=V_{c} / n .
$$

\section{Thus}

$$
\begin{aligned}
& m=(d P / d T)_{V}=\left[\frac{\pi}{\left(V_{c}-b\right)}\right. \\
& +\frac{B_{2}}{\left(V_{c}-b\right)^{2}}+\frac{B_{3}}{\left(V_{c}-b\right)^{3}} \\
& \left.+\frac{B_{5}}{\left(V_{c}-b\right)^{5}}\right]-\left[\frac{C_{2}}{\left(V_{c}-b\right)^{2}}\right. \\
& \left.+\frac{C_{3}}{\left(V_{c}-b\right)^{3}}+\frac{C_{5}}{\left(V_{c}-b\right)^{5}}\right] \\
& \quad \frac{k \exp \left(-k T / T_{c}\right)}{T_{c}}
\end{aligned}
$$

and

$$
\begin{aligned}
& m y=\left(d P / d T^{\prime}\right)_{V}=\left[\frac{R}{\left(V_{c} / n-b\right)}\right. \\
& +\frac{B_{2}}{\left(V_{c} / n-b\right)^{2}}+\frac{B_{3}}{\left(V_{d} / n-b\right)^{3}} \\
& \left.+\frac{B_{5}}{\left(V_{c} / n-b\right)^{5}}\right]-\left[\frac{C_{2}}{\left(V_{c} / n-b\right)^{2}}\right.
\end{aligned}
$$


Table 1. Constants for the Equations of State for Carbon. Dioxide

\begin{tabular}{|c|c|c|}
\hline & $\begin{array}{l}\text { Original } \\
\text { equation }\end{array}$ & $\begin{array}{l}\text { Modified } \\
\text { equation }\end{array}$ \\
\hline$T_{c}$ & $547.5^{\circ} \mathrm{R}$ & Same \\
\hline$P_{c}$ & $\begin{array}{l}1069.4 \text { lb./sq. in. } \\
\text { abs. }\end{array}$ & Same \\
\hline$V_{c}$ & $0.03454 \mathrm{cu} . \mathrm{ft} . / \mathrm{lb}$ & Same \\
\hline$Z_{\mathrm{c}}$ & 0.27671 & Same \\
\hline$m$ & 14.0 & Same \\
\hline$\beta$ & 3.25 & Same \\
\hline$T_{b} / T_{c}$ & 2.3 & Same \\
\hline$T^{\prime} / T_{c}$ & 0.80 & Same \\
\hline$n$ & $\ldots$ & 1.70 \\
\hline$y$ & & 3.50 \\
\hline$b$ & 0.007495 & Same \\
\hline$A_{2}$ & -8.9273631 & Same \\
\hline$A_{3}$ & 0.18907819 & 0.17948621 \\
\hline$A_{4}$ & $-2.112459 \times 10^{-3}$ & Same \\
\hline & & $7.017835 \times 10^{-6}$ \\
\hline$B_{1}(R)$ & 0.24381 & Same \\
\hline$B_{2}$ & $5.262476 \times 10^{-\mathrm{a}}$ & Same \\
\hline$B_{8}$ & $-7.04617 \times 10^{-5}$ & $-5.770542 \times 10^{-}$ \\
\hline$B_{5}$ & $1.9565593 \times 10^{-8}$ & $1.023511 \times 10^{-8}$ \\
\hline$C_{2}$ & -150.97587 & Same \\
\hline$C_{3}$ & $4.0831424^{*}$ & 4.705805 \\
\hline & $\ldots$ & $-4.55437 \times 10^{-4}$ \\
\hline
\end{tabular}

*Misprinted in ( 1 ) as 0.0831424 .

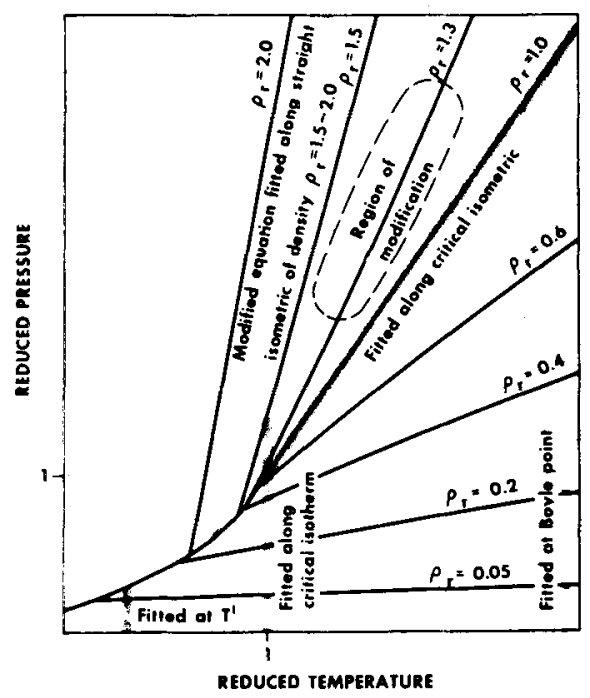

Fig. 1. Typical isometric plot.

$$
\begin{gathered}
\left.+\frac{C_{3}}{\left(V_{c} / n-b\right)^{3}}+\frac{C_{5}}{\left(V_{c} / n-b\right)^{5}}\right] \\
\frac{k \exp \left(-k T / T_{c}\right)}{T_{c}}
\end{gathered}
$$

The elimination of $B_{5}$ from Equations (11) and (12) yields

$$
\begin{gathered}
B_{3}=\frac{m\left(V_{c}-b\right)^{5}-m y\left(V_{c} / n-b\right)^{5}}{\left(V_{c}-b\right)^{2}-\left(V_{c} / n-b\right)^{2}} \\
-\frac{B_{2}\left(V_{c}-b\right)^{3}+B_{2}\left(V_{c} / n-b\right)^{3}}{\left(V_{c}-b\right)^{2}-\left(V_{c} / n-b\right)^{2}} \\
-R\left[\left(V_{c}-b\right)^{2}+\left(V_{c} / n-b\right)^{2}\right]
\end{gathered}
$$

Correspondingly the elimination of $B_{3}$ results in

$B_{5}=m\left(V_{c}-b\right)^{5}-R\left(V_{c}-b\right)^{4}$

- $B_{2}\left(V_{c}-b\right)^{3}-B_{3}\left(V_{c}-b\right)^{2}$

The two remaining $A$ 's may be solved for by substitution into Equation (2). Thus

$$
\begin{aligned}
& A_{3}=f_{3}\left(T_{c}\right)-B_{3} T_{c} \\
& -C_{3} \exp (-k) \\
& -C_{5} \exp (-k)
\end{aligned}
$$

The complete expanded form of the modified equation containing the new constants $A_{5}$ and $C_{5}$ is

$$
\begin{aligned}
P & =\frac{R T}{V-b} \\
& +\frac{A_{2}+B_{2} T+C_{2} \exp \left(-k T / T_{c}\right)}{(V-b)^{2}} \\
& +\frac{A_{3}+B_{3} T+C_{3} \exp \left(-k T / T_{c}\right)}{(V-b)^{3}} \\
& +\frac{A_{4}}{(V-b)^{4}} \\
& +\frac{A_{5}+B_{5} T+C_{3} \exp \left(-k T / T_{c}\right)}{(V-b)^{5}}
\end{aligned}
$$

For testing the improvement of the modified equation over the original equation, the highly precise data for carbon dioxide were chosen. In the accompanying table are listed the equation constants calculated for carbon dioxide, both for the original equation and for the modified equation. Using these constants in Equation (3) and (17) the authors computed the pressures for several hundred points at varying temperatures and densities. The computed pressures were compared with the experimental pressures reported by Michels and deGroot (2). The important points which may be drawn from this comparison are

1. The modification changed the predicted pressures insignificantly for densities less than the critical density. In this range the pressures predicted by the two equations are never more than $0.25 \%$ apart. Also neither equation disagrees with the experimental data by more than $0.50 \%$, and the average error is less than $0.20 \%$.

2. At densities greater than 1.3 times the critical density and temperatures above the critical, the original equation predicts pressures which dip below the measured pressures and then rise above them at the highest temperatures. For example, at 1.50 times the critical density the original equation predicts pressures which, over a $180^{\circ} \mathrm{R}$. temperature range, go from $1.30 \%$ greater than the measured pressure, to $6.32 \%$ less, and then to $5.50 \%$ greater. The modified equation substantially corrects this error. For the same example at 1.50 times the critical density, pressures are predicted which go uniformly from $0.17 \%$ greater than the experimental to $5.25 \%$ greater. At this density the proposed equation is more accurate than the original equation $(2.02 \%$ average error vs. $2.92 \%)$.

3. In the density range from 1.2 to 1.4 times the critical density the modified equation is much more aceurate than the original equation. The modified equation has a maximum error of $1.68 \%$ in this range compared with $5.98 \%$ for the original equation and an average error of $0.93 \%$ vs. $2.68 \%$ for the original equation. In this region the modified equation also has more accurate derivatives of pressure with respect to temperature than the original equation.

\section{CONCLUSIONS}

The data show that the modified equation represents the experimental data with greater precision than the original equation in the neighborhood of 1.4 times the critical density. The modification makes the equation a more useful tool for thermodynamic calculations which require precise relations between pressure, volume, and temperature.

\section{NOTATION}

$A_{1}, B_{1}, C_{1}, A_{2}, \cdots=$ characteristic constants in Equation (2)

$M=$ slope of reduced vapor pressure curve at critical point $d P_{r} / d T_{\tau}=$ $-m P_{c} / T_{c}$

$P=$ pressure

$R=$ universal gas constant

$T=$ absolute temperature

$T_{b}=$ Boyle point temperature

$T^{\prime}=$ absolute temperature for which the slope at $P=0$ of the isotherm on the compressibility chart equals the slope of the line joining the critical point and $(Z=1, P=0)$

$V=$ specific volume or molal volume

$Z=$ compressibility factor $=P V / R T$

$b=$ characteristic constant for each substance

$f_{1}, f_{2} \ldots=$ temperature functions defined by Equation (2)

$f_{1}\left(T_{c}\right), f_{2}\left(T_{c}\right) \cdots=$ preceding functions evaluated at $T=T$.

$k=$ constant

$m=$ slope of the critical isometric on a pressure-temperature diagram

$n=$ reduced density $>1.0$ at which $\left(d^{2} P / d T^{3}\right)_{V}=0$

$y=$ ratio of slope of isometric of reduced density $n$ to slope of critical isometric

$\beta=$ characteristic constant in $V_{c}-b=$ $\beta V_{c} / 15 Z_{0}$

$\rho=$ density

\section{Subseripts}

$c=$ value at critical point, for example, $T_{c}, P_{c}$

$i=$ summation index

$r=$ reduced property, $T_{r}=T / T_{c}$

\section{LITERATURE CITED}

1. Martin, J. J., and Y. C. Hou, A.I.Ch.E. Journal, 1, 142 (June, 1955).

2. Michels, A., and S. R. deGroot, Appl. Sci. Research, A, 1, 565 (1948).

Manuscript reeeived November 11, 1957; revision received August 22; 1958 ; paper accepted August 26, 1958 . 